Бюллетень Почвенного института им. В.В. Докучаева. 2021. Вып. 108

Dokuchaev Soil Bulletin, 2021, 108

UDC 631.4

(c) (1)

DOI: $10.19047 / 0136-1694-2021-108-104-136$

Check for updates

Cite this article as:

Nurdin, Rayes M.L., Soemarno, Sudarto, Listyarini E., Agustina C., Rahman R., Rauf A., Husain J., Soils in the Bulia micro watershed of Gorontalo province, Indonesia, and their quality assessment, Dokuchaev Soil Bulletin, 2021, V. 108, pp. 104-136, DOI: 10.19047/0136-1694-2021-108-104-136

\title{
Acknowledgments:
}

We would like to pay our regards to the Faculty of Agriculture, Brawijaya University who has funded this research with a non-tax state revenue grant in accordance with the Budget Implementation List Number: DIPA042.01.2.400919/2019.

\section{Soils in the Bulia micro watershed of Gorontalo province, Indonesia, and their quality assessment}

(C) 2021 Nurdin $^{1 *}$, M. L. Rayes ${ }^{2}$, Soemarno ${ }^{2}$, Sudarto ${ }^{2}$, E. Listyarini ${ }^{2}$, C. Agustina ${ }^{2}$, R. Rahman ${ }^{1}$, A. Rauf ${ }^{3}$, J. Husain ${ }^{4}$

${ }^{I}$ Department of Agrotechnology, Gorontalo State University,

Prof. Dr. Ing. B. J. Habibie Str., Moutong Village,

96554 Bone Bolango, Indonesia,

"https://orcid.org/0000-0001-5725-5694, e-mail: nurdin@ung.ac.id.

${ }^{2}$ Department of Soil Science, Brawijaya University,

No. 54 Veteran Str., Lowokwaru, 65145 Malang, Indonesia.

${ }^{3}$ Department of Agribussiness, Gorontalo State University,

Prof. Dr. Ing. B. J. Habibie Str., Moutong Village, 96554 Bone Bolango, Indonesia.

${ }^{4}$ Department of Soil Science, Sam Ratulangi University, No. 1 Kleak Bahu Str., Kleak, 95115 Manado, Indonesia.

Received 12.07.2021, Revised 24.08.2021, Accepted 20.09.2021

Abstract: Ten representative pedons from the Bulia micro watershed of Gorontalo Province, Indonesia, were characterized and classified to determine its land quality (LQ) class. Angular blocky, sticky, plastic consistencies and a hard consistency prevailed in the soil structure. In the alluvial plains the soil texture is dominated by the clay fraction, while in the hills and volcanic mountains the sand fraction is dominated. The soils in the Bulia micro watershed also have acid to neutral reaction, with the range of very low to 
high OC (organic carbon) levels, the reserve of exchangeable bases was dominated by $\mathrm{Ca}^{2+}$ in two series patterns, namely: $\mathrm{Ca}^{2+}>\mathrm{Mg}^{+}>\mathrm{Na}^{+}>\mathrm{K}^{+}$and $\mathrm{Ca}^{2+}>\mathrm{Na}+>\mathrm{Mg}^{+}>\mathrm{K}^{+}$, cation exchange capacity (CEC) ranged from low to very high, and the base saturation varied from moderate to very high. The alluvial plain is represented by Inceptisol in P1 and Typic Humustepts (P7), also by Oxic Humustepts (P3), then Mollisol on P4 (Typic Argiudolls) and Typic Haplustolls (P6), Alfisol on P5 (Typic Paleustalfs). Entisol on P2 (Typic Ustipsamments) was found in volcanic mountains and P9 (Typic Paleustolls) P8 (Ultic Paleustalfs), P10 (Inceptic Haplustalfs) are typical of volcanic hills. On the alluvial plains the land was categorized as the LQ class II, III and IV, the volcanic mountains were the LQ class IV, while the land on the volcanic hills was categorized as the LQ class VI. River bank erosion on the land river terraces can be held by the manufacture of gabions, talud, cliff reinforcement plants and terraces. The soil temperatures and high clay content can be regulated by mulching and organic materials.

Keywords: Characterization, classification, soils of Indonesia, land quality, the Bulia watershed.

\section{INTRODUCTION}

Land is a crucial component of land resources which enables plants growth and food production. Land and plant productivity is primarily determined by soil and other land characteristics related to land quality (Subardja, Sudarsono, 2005). However, an intense tillage during agricultural cultivation and a pressure of the land use when its conservation and sustainability are ignored has resulted into a decrease of land quality. Agricultural production tends to level or even decrease (Nurdin, 2012). Corn is a traditional commercial crop in the province of Gorontalo, Indonesia, which has been intensively and massively cultivated since it was established as a prime commodity in the agropolitan program in 2001. Until 2019, hybrid corn yields in Gorontalo Province reached 1.7 million tons or increased by $9.3 \%$ compared to 2018 , however, the productivity of maize was still low at only 5.0 tons/ha (BPS Gorontalo Province, 2020). In fact, the potential corn yield in Indonesia can reach 10-11 tons/ha (Yasin et al., 2015), while the achievement of the national productivity in 2018 was only 5.2 tons/ha (Indonesian Ministry of Agriculture, 2019).

The Bulia micro watershed area is a corn production centre that also supports the agricultural area below. The watershed has a vital role 
because it supplies irrigation water for agriculture and other activities (Mahapatra et al., 2019). The corn cultivation in this watershed has exceeded the carrying capacity indicated for the corn planting on the slope of $>25 \%$, so that the land degradation often occurs. Soil erosion, according to the corn agropolitan program of Gorontalo, reached 1,396 tons/ha/year (Husain et al., 2004). Meanwhile, the corn productivity in this area is only 5.0 tons/ha (BPS Gorontalo Regency, 2020), which could happen due to the fact that the crop was cultivated on a nonsuitable land.

Soil characterization is essential because it provides some necessary information about the soil characteristics for growing plants (Devi et al., 2015). For sustainable management of soil resources in agroecological areas we need timely monitoring of significant physical, chemical and biological soil characteristics and responses to the changes in land management (Supriya et al., 2019). These soil characteristics then form the basis for land classification. Combining soil characterization with classification is a powerful tool to develop management strategies for food security and environmental sustainability (Satish et al., 2018). However, the efforts to link land characteristics and classification with a specific land quality are still relatively rare. Land quality is a land ability to perform a specific function before the land is degraded (Beinroth et al., 2001). Understanding soil types and their distribution, its limits and potential is essential for a proper management to increase productivity and yields (Niranjana et al., 2011).

The survey and mapping of soil resources in the Paguyaman watershed were carried out by the Soil and Agro-climate Research Centre of the Indonesian Ministry of Agriculture (Puslittanak Research Team, 1995), however, the mapping scale was $1: 50,000$. In 2010, some research was carried out on the development, classification and potential of the paddy soils on toposequence (Nurdin, 2010), however, it only focused on the rainfed paddy soils, while the dry land was only compared to the locations close to the soil pedon in the rice fields. Considering the high intensity of the land management and the massive corn cultivation in this sub-watershed, this research has become significant.

\section{MATERIALS AND METHODS}

Study location 
The Bulia micro watershed is a part of the Paguyaman watershed located in the northern part, it covers Mootilango and Boliyohuto District of Gorontalo Regency of Gorontalo Province, Indonesia (Figure 1). Geographically, the research location is between $0^{\circ} 39^{\prime} 123^{\prime \prime}$ and $0^{\circ} 51^{\prime} 321^{\prime \prime} \mathrm{N}, 122^{\circ} 35^{\prime} 21^{\prime \prime}$ and 122 $43^{\prime} 12^{\prime \prime} \mathrm{S}$ (Table 1), which is $67 \mathrm{~km}$ from Gorontalo City, Indonesia.

Table 1. Site characteristics of the pedons in the Bulia micro watershed

\begin{tabular}{|l|l|c|l|c|l|}
\hline \multicolumn{1}{|c|}{$\begin{array}{c}\text { Pedon/ } \\
\text { Villages }\end{array}$} & \multicolumn{1}{|c|}{ Location } & $\begin{array}{c}\text { Elevation } \\
\text { (m msl) }\end{array}$ & Landform & $\begin{array}{c}\text { Slope } \\
(\%)\end{array}$ & Drainage \\
\hline $\begin{array}{l}\text { P1, } \\
\text { Tolite }\end{array}$ & $\begin{array}{l}0^{\circ} 39^{\prime} 44.80^{\prime \prime} \mathrm{N} \\
122^{\circ} 35^{\prime} 27.20^{\prime \prime} \mathrm{S}\end{array}$ & 24 & $\begin{array}{l}\text { Alluvial } \\
\text { plain }\end{array}$ & $0-3$ & $\begin{array}{l}\text { Poorly } \\
\text { drained }\end{array}$ \\
\hline $\begin{array}{l}\text { P2, } \\
\text { Monggolito }\end{array}$ & $\begin{array}{l}0^{\circ} 40^{\prime} 01.20^{\prime \prime} \mathrm{N} \\
122^{\circ} 37^{\prime} 57.20^{\prime \prime} \mathrm{S}\end{array}$ & 159 & $\begin{array}{l}\text { Volcanic } \\
\text { mountain }\end{array}$ & $15-30$ & $\begin{array}{l}\text { Well } \\
\text { drained }\end{array}$ \\
\hline $\begin{array}{l}\text { P3, } \\
\text { Huyula }\end{array}$ & $\begin{array}{l}0^{\circ} 42^{\prime} 59.9^{\prime \prime} \mathrm{N} \\
122^{\circ} 39^{\prime} 43.2^{\prime \prime} \mathrm{S}\end{array}$ & 63 & $\begin{array}{l}\text { Alluvial } \\
\text { plain }\end{array}$ & $0-3$ & $\begin{array}{l}\text { Moderately } \\
\text { drained }\end{array}$ \\
\hline $\begin{array}{l}\text { P4, } \\
\text { Payu }\end{array}$ & $\begin{array}{l}0^{\circ} 44^{\prime} 04.4^{\prime \prime} \mathrm{N} \\
122^{\circ} 37^{\prime} 48.4^{\prime \prime} \mathrm{S}\end{array}$ & 53 & $\begin{array}{l}\text { Alluvial } \\
\text { plain }\end{array}$ & $0-3$ & $\begin{array}{l}\text { Moderately } \\
\text { drained }\end{array}$ \\
\hline $\begin{array}{l}\text { P5, } \\
\text { Pilomonu }\end{array}$ & $\begin{array}{l}0^{\circ} 43^{\prime} 53.80^{\prime \prime} \mathrm{N} \\
122^{\circ} 35^{\prime} 22.60^{\prime \prime} \mathrm{S}\end{array}$ & 75 & $\begin{array}{l}\text { Alluvial } \\
\text { plain }\end{array}$ & $0-3$ & $\begin{array}{l}\text { Moderately } \\
\text { drained }\end{array}$ \\
\hline $\begin{array}{l}\text { P6, } \\
\text { Karyamukti }\end{array}$ & $\begin{array}{l}0^{\circ} 42^{\prime} 20.50^{\prime \prime} \mathrm{N} \\
122^{\circ} 41^{\prime} 05.50^{\prime \prime} \mathrm{S}\end{array}$ & 109 & $\begin{array}{l}\text { Alluvial } \\
\text { plain }\end{array}$ & $3-8$ & $\begin{array}{l}\text { Moderately } \\
\text { drained }\end{array}$ \\
\hline $\begin{array}{l}\text { P7, } \\
\text { Karyamukti }\end{array}$ & $\begin{array}{l}0^{\circ} 42^{\prime} 10.30^{\prime \prime} \mathrm{N} \\
122^{\circ} 41^{\prime} 19.40^{\prime \prime} \mathrm{S}\end{array}$ & 114 & $\begin{array}{l}\text { Alluvial } \\
\text { plain }\end{array}$ & $3-8$ & $\begin{array}{l}\text { Moderately } \\
\text { drained }\end{array}$ \\
\hline $\begin{array}{l}\text { P8, } \\
\text { Sukamaju }\end{array}$ & $\begin{array}{l}0^{\circ} 44^{\prime} 05^{\prime \prime} \mathrm{N} \\
122^{\circ} 40^{\prime} 04^{\prime \prime} \mathrm{S}\end{array}$ & 253 & $\begin{array}{l}\text { Volcanic } \\
\text { hill }\end{array}$ & $8-15$ & $\begin{array}{l}\text { Well } \\
\text { drained }\end{array}$ \\
\hline $\begin{array}{l}\text { P9, } \\
\text { Payu }\end{array}$ & $\begin{array}{l}0^{\circ} 45^{\prime} 12^{\prime \prime} \mathrm{N} \\
122^{\circ} 38^{\prime} 08^{\prime \prime} \mathrm{S}\end{array}$ & 285 & $\begin{array}{l}\text { Volcanic } \\
\text { hill }\end{array}$ & $8-15$ & $\begin{array}{l}\text { Well } \\
\text { drained }\end{array}$ \\
\hline $\begin{array}{l}\text { P10, } \\
\text { Huyula }\end{array}$ & $\begin{array}{l}0^{\circ} 43^{\prime} 11.10^{\prime \prime} \mathrm{N} \\
122^{\circ} 40^{\prime} 31.20^{\prime \prime} \mathrm{S}\end{array}$ & 262 & $\begin{array}{l}\text { Volcanic } \\
\text { hill }\end{array}$ & $8-15$ & $\begin{array}{l}\text { Well } \\
\text { drained }\end{array}$ \\
\hline
\end{tabular}

Overall, the Bulia micro watershed has $21,456.58$ ha and consists of upland amounted to $18,993.44$ ha $(32.59 \%)$, and paddy fields amounted to $2,991.15$ ha (13.94\%). Specifically, the upland agriculture covers the agricultural land areas amounted to 6,993.44 ha (37.87\%), settlement areas -461.59 ha $(2.50 \%)$, and forest areas amounted to $11,010.40$ ha $(59.63 \%)$. The soils in this area are generally developed from volcanic material in the upper watershed and lacustrine deposits in the middle and the bottom of the watershed. The study area is locat- 
ed in tropical climate with rainy and dry seasons. The average annual rainfall was only $1,478 \mathrm{~mm}$ with 1 wet month only and 4 dry months, so it belongs to the E2 agro-climate zone (Oldeman, Darmiyati, 1977). The average annual air temperature reaches $28.19{ }^{\circ} \mathrm{C}$ with the maximum temperature of $28.73{ }^{\circ} \mathrm{C}$ and the minimum temperature of $27.63^{\circ} \mathrm{C}$. Under these conditions, the soil moisture regime is determined ustic and the soil temperature regime - isohyperthermic (Soil Survey Staff, 2014).

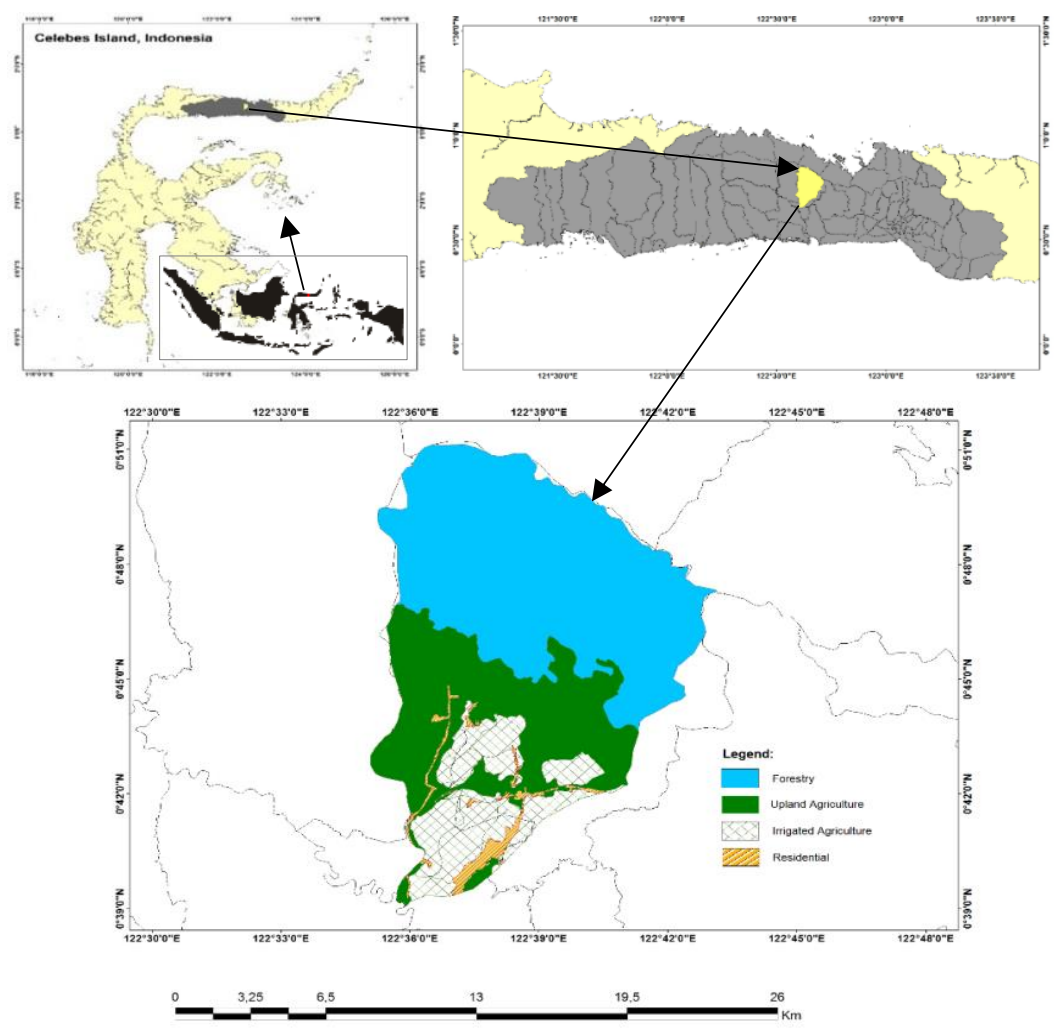

Fig 1. Location Map of the Bulia Micro watershed. 
Soil surveying, characterization and classification

Ten representative pedons were selected to conduct the soil survey. The description of soil morphology refers to the Soil Survey Manual (Soil Science Manual, 2017; Sukarman et al., 2017). According to standard procedures, soil samples were taken at each horizon and their physical and chemical properties were analyzed (Jackson, 1973; Eviyati, Sulaeman, 2009). The morphological and the soil properties data obtained during the laboratory analysis are used for the soil characterization together with the climate and the terrain conditions data, according to Sukarman et al. (2017). The soil characteristics are used for soil classification according to the keys to soil taxonomy (Soil Survey Staff, 2014).

\section{Land quality assessment}

Land quality (LQ) class assessment follows the method according to Beinroth et al. (2001). This method has been modified based on availability of soil characteristics and classification data without including local population data. Soil pedons (P), which were classified in taxa according to the Soil Taxonomy System (Soil Survey Staff, 2014), were combined with a land unit (LU) basing on the similarity of the criteria in taxa. Soil and pedoclimate information was used to place each LU into I to IX land quality classes with class I having the most appropriate attributes and class IX having the least suitable ones for crop production. The results of the land quality analysis are widely presented and described with the help of Arc GIS.

\section{RESULTS AND DISCUSSION}

\section{Morphology and soil physical properties}

The results of field studies and the laboratory characterization of ten soil pedons were presented in Table 2, Figure 2. The soils in the study area have been developed as indicated by the horizon structuring (horizons A and B), with the depth of the soil solum varying from shallow to very deep. The soil colour is only of 7.5YR and 10YR hue, where 7.5YR is dominant. P1, P2, P3, P5, P8, P9 and P10 soil colour varies from dark brown, brown to strong brown with hue $7.5 \mathrm{YR}$, ranging from 3 to 5 , and chroma - from 1 to 6 . 
Table 2. Morphological characteristics and soil physical properties in the Bulia micro watershed

\begin{tabular}{|c|c|c|c|c|c|c|c|c|c|c|}
\hline \multirow{2}{*}{$\begin{array}{c}\begin{array}{c}\text { Pedon } \\
\text { and } \\
\text { Horizon }\end{array} \\
\end{array}$} & \multirow{2}{*}{$\begin{array}{l}\text { Depth } \\
\text { (cm) }\end{array}$} & \multirow{2}{*}{$\begin{array}{c}\text { Colour } \\
\text { Moisture }\end{array}$} & \multirow{2}{*}{$\begin{array}{l}\text { Struc- } \\
\text { tures }\end{array}$} & \multicolumn{3}{|c|}{ Consistence } & Sand & Silt & Clay & \multirow{2}{*}{$\begin{array}{c}\text { Texture } \\
\text { Class }\end{array}$} \\
\hline & & & & $\mathbf{w}$ & $\mathbf{m}$ & $\mathbf{D}$ & \multicolumn{3}{|c|}{$\%$} & \\
\hline \multicolumn{11}{|c|}{ P1 (Alluvial Plain) } \\
\hline Ap & $0-23$ & $7.5 \mathrm{YR} 4 / 4$ & $\mathrm{~m}, 3$, abk & $\mathrm{s}, \mathrm{p}$ & fi & $\mathrm{H}$ & 9 & 40 & 51 & Silty Clay \\
\hline Bw1 & $23-43$ & $7.5 \mathrm{YR} 4 / 2$ & $\mathrm{~m}, 3$, abk & ss, sp & fi & $\mathrm{H}$ & 3 & 46 & 51 & Silty Clay \\
\hline $\mathrm{Bw} 2$ & $43-75$ & $7.5 \mathrm{YR} 4 / 4$ & $\mathrm{~m}, 3$, abk & ss, sp & fi & $\mathrm{H}$ & 3 & 54 & 43 & Silty Clay \\
\hline Bw3 & $75-100$ & $7.5 \mathrm{YR} 4 / 2$ & $\mathrm{~m}, 1$, abk & ss, sp & vfi & $\mathrm{Vh}$ & 3 & 46 & 51 & Silty Clay \\
\hline \multicolumn{11}{|c|}{ P2 (Volcanic Mountains) } \\
\hline Ap & $0-5$ & $7.5 \mathrm{YR} 4 / 4$ & $\mathrm{f}, 1, \mathrm{cr}$ & so, po & fr & $\mathrm{L}$ & 85 & 10 & 5 & Loamy sand \\
\hline Bw1 & $5-37$ & $7.5 \mathrm{YR} 4 / 4$ & $\mathrm{f}, 2, \mathrm{sbk}$ & so, po & $\mathrm{fr}$ & $\mathrm{L}$ & 84 & 2 & 14 & Loamy sand \\
\hline $\mathrm{Bw} 2$ & $37-61$ & $7.5 \mathrm{YR} 4 / 4$ & $\mathrm{~m}, 2, \mathrm{p}$ & so, po & fr & $\mathrm{L}$ & 75 & 10 & 15 & Sandy Loam \\
\hline $\mathrm{C}$ & $61+$ & $7.5 \mathrm{YR} 4 / 2$ & $\mathrm{~m}, 3, \mathrm{p}$ & so, po & fr & $\mathrm{L}$ & 66 & 15 & 19 & Sandy Loam \\
\hline \multicolumn{11}{|c|}{ P3 (Alluvial Plain) } \\
\hline Ap & $0-14$ & $7.5 \mathrm{YR} 3 / 3$ & $\mathrm{f}, 1, \mathrm{cr}$ & ss, sp & fi & $\mathrm{H}$ & 33 & 41 & 26 & Loam \\
\hline Bw1 & $14-43$ & $7.5 \mathrm{YR} 4 / 4$ & $\mathrm{f}, 2, \mathrm{abk}$ & $\mathrm{s}, \mathrm{p}$ & fi & $\mathrm{H}$ & 31 & 37 & 32 & Clay Loam \\
\hline $\mathrm{Bw} 2$ & $43-68$ & $7.5 \mathrm{YR} 4 / 4$ & f, 1, abk & ss, sp & fi & $\mathrm{H}$ & 23 & 54 & 23 & Silty Loam \\
\hline Bw3 & $68+$ & 10 YR 3/6 & $\mathrm{f}, 1, \mathrm{cr}$ & $\mathrm{s}, \mathrm{p}$ & fi & $\mathrm{H}$ & 23 & 48 & 29 & Clay Loam \\
\hline
\end{tabular}




\begin{tabular}{|c|c|c|c|c|c|c|c|c|c|c|}
\hline \multirow{2}{*}{$\begin{array}{c}\begin{array}{c}\text { Pedon } \\
\text { and } \\
\text { Horizon }\end{array} \\
\end{array}$} & \multirow{2}{*}{$\begin{array}{l}\text { Depth } \\
(\mathrm{cm})\end{array}$} & \multirow{2}{*}{$\begin{array}{l}\text { Colour } \\
\text { Moisture }\end{array}$} & \multirow{2}{*}{$\begin{array}{l}\text { Struc- } \\
\text { tures }\end{array}$} & \multicolumn{3}{|c|}{ Consistence } & \multirow{2}{*}{ Sand } & \multirow{2}{*}{ Silt } & \multirow{2}{*}{ Clay } & \multirow{2}{*}{$\begin{array}{l}\text { Texture } \\
\text { Class }\end{array}$} \\
\hline & & & & $\mathbf{w}$ & $\mathbf{m}$ & D & & & & \\
\hline \multicolumn{11}{|c|}{ P4 (Alluvial Plain) } \\
\hline Ap & $0-14$ & $10 \mathrm{YR} 3 / 3$ & $\mathrm{~m}, 3$, abk & $\mathrm{s}, \mathrm{p}$ & fi & $\mathrm{H}$ & 27 & 26 & 47 & Clay \\
\hline $\mathrm{Bw}$ & $14-50$ & $10 \mathrm{YR} 4 / 3$ & $\mathrm{f}, 1, \mathrm{abk}$ & $\mathrm{s}, \mathrm{p}$ & fi & $\mathrm{H}$ & 32 & 34 & 34 & Clay Loam \\
\hline Bt1 & $50-81$ & $10 \mathrm{YR} 3 / 2$ & f, 1 , abk & ss, sp & fi & $\mathrm{H}$ & 12 & 24 & 64 & Clay \\
\hline $\mathrm{Bt} 2$ & $81+$ & $7.5 \mathrm{YR} 4 / 1$ & $\mathrm{f}, 1, \mathrm{abk}$ & $\mathrm{s}, \mathrm{p}$ & fi & $\mathrm{H}$ & 16 & 25 & 59 & Clay \\
\hline \multicolumn{11}{|c|}{ P5 (Alluvial Plain) } \\
\hline Ap & $0-21$ & $7.5 \mathrm{YR} 4 / 4$ & $\mathrm{f}, 2$, sbk & ss, sp & fi & $\mathrm{H}$ & 23 & 43 & 34 & Clay Loam \\
\hline $\mathrm{Bw}$ & $21-46$ & $7.5 \mathrm{YR} 4 / 4$ & f, 2, sbk & ss, sp & fi & $\mathrm{H}$ & 23 & 48 & 29 & Clay Loam \\
\hline Bt1 & $46-84$ & $7.5 \mathrm{YR} 4 / 3$ & $\mathrm{f}, 2, \mathrm{abk}$ & $\mathrm{s}, \mathrm{p}$ & fi & $\mathrm{H}$ & 25 & 35 & 40 & Clay \\
\hline $\mathrm{Bt} 2$ & $84-117$ & $7.5 \mathrm{YR} 4 / 6$ & $\mathrm{~m}, 2, \mathrm{p}$ & $\mathrm{s}, \mathrm{p}$ & fi & $\mathrm{H}$ & 24 & 36 & 40 & Clay \\
\hline Btg & $117+$ & 7.5 YR 5/8 & $\mathrm{m}, 1, \mathrm{sbk}$ & ss, sp & fi & $\mathrm{H}$ & 8 & 46 & 46 & Silty Clay \\
\hline \multicolumn{11}{|c|}{ P6 (Alluvial Plain) } \\
\hline Ap & $0-12$ & $10 \mathrm{YR} 3 / 3$ & $\mathrm{f}, 1, \mathrm{abk}$ & $\mathrm{s}, \mathrm{p}$ & fi & $\mathrm{H}$ & 84 & 15 & 1 & Loamy Sand \\
\hline Bw1 & $12-34$ & $10 \mathrm{YR} 3 / 4$ & f, 1 , abk & $\mathrm{s}, \mathrm{p}$ & fi & $\mathrm{H}$ & 61 & 10 & 29 & $\begin{array}{c}\text { Sandy Clay } \\
\text { Loam }\end{array}$ \\
\hline Bw2 & $34-71$ & $10 \mathrm{YR} 4 / 6$ & $\mathrm{~m}, 3$, abk & ss, sp & fi & $\mathrm{H}$ & 61 & 24 & 15 & Sandy Loam \\
\hline $\mathrm{C}$ & $71-90$ & 7.5 YR 5/8 & $\mathrm{f}, 1, \mathrm{cr}$ & so, po & fr & $\mathrm{L}$ & 84 & 5 & 11 & Loamy Sand \\
\hline
\end{tabular}




\begin{tabular}{|c|c|c|c|c|c|c|c|c|c|c|}
\hline \multirow{2}{*}{$\begin{array}{c}\text { Pedon } \\
\text { and } \\
\text { Horizon }\end{array}$} & \multirow{2}{*}{$\begin{array}{l}\text { Depth } \\
\text { (cm) }\end{array}$} & \multirow{2}{*}{$\begin{array}{l}\text { Colour } \\
\text { Moisture }\end{array}$} & \multirow{2}{*}{$\begin{array}{l}\text { Struc- } \\
\text { tures }\end{array}$} & \multicolumn{3}{|c|}{ Consistence } & \multirow{2}{*}{ Sand } & \multirow{2}{*}{ Silt } & \multirow{2}{*}{ Clay } & \multirow{2}{*}{$\begin{array}{l}\text { Texture } \\
\text { Class }\end{array}$} \\
\hline & & & & $\mathbf{w}$ & $\mathbf{m}$ & D & & & & \\
\hline \multicolumn{11}{|c|}{ P7 (Alluvial Plain) } \\
\hline Ap & $0-6$ & $7.5 \mathrm{YR} 4 / 6$ & $\mathrm{~m}, 1, \mathrm{abk}$ & $\mathrm{s}, \mathrm{p}$ & fi & $\mathrm{H}$ & 33 & 11 & 56 & Clay \\
\hline Bw1 & $6-17$ & $10 \mathrm{YR} 4 / 6$ & $\mathrm{~m}, 3$, sbk & $\mathrm{s}, \mathrm{p}$ & fi & $\mathrm{H}$ & 29 & 20 & 51 & Clay \\
\hline Bw2 & $17-33$ & $10 \mathrm{YR} 3 / 6$ & $\mathrm{~m}, 1, \mathrm{abk}$ & ss, sp & fi & $\mathrm{H}$ & 21 & 20 & 59 & Clay \\
\hline $\mathrm{Bt}$ & $33-49$ & $10 \mathrm{YR} 3 / 6$ & $\mathrm{f}, 1, \mathrm{p}$ & ss, sp & fi & $\mathrm{H}$ & 19 & 15 & 66 & Clay \\
\hline $\mathrm{BC}$ & $49+$ & $7.5 \mathrm{YR} 4 / 6$ & $\mathrm{f}, 1, \mathrm{abk}$ & ss, sp & fi & $\mathrm{H}$ & 18 & 29 & 53 & Clay \\
\hline \multicolumn{11}{|c|}{ P8 (Volcanic Hills) } \\
\hline Ap & $0-7$ & $7.5 \mathrm{YR} 3 / 3$ & $\mathrm{f}, 1, \mathrm{abk}$ & ss, sp & fi & $\mathrm{H}$ & 64 & 10 & 26 & $\begin{array}{c}\text { Sandy Clay } \\
\text { Loam }\end{array}$ \\
\hline $\mathrm{Bw}$ & $7-24$ & $7.5 \mathrm{YR} 3 / 3$ & $\mathrm{~m}, 1$, abk & $\mathrm{s}, \mathrm{p}$ & fi & $\mathrm{H}$ & 47 & 24 & 29 & $\begin{array}{c}\text { Sandy Clay } \\
\text { Loam }\end{array}$ \\
\hline Bt1 & $24-44$ & $7.5 \mathrm{YR} 4 / 6$ & $\mathrm{f}, 3, \mathrm{p}$ & $\mathrm{s}, \mathrm{p}$ & fi & $\mathrm{H}$ & 45 & 15 & 40 & Sandy Clay \\
\hline Bt2 & $44-63$ & 7.5 YR 5/6 & $\mathrm{m}, 3$, abk & $\mathrm{s}, \mathrm{p}$ & fi & $\mathrm{H}$ & 42 & 16 & 42 & Clay \\
\hline $\mathrm{Bt} 3$ & $63+$ & 7.5 YR 5/6 & $\mathrm{m}, 1, \mathrm{abk}$ & $\mathrm{s}, \mathrm{p}$ & fi & $\mathrm{H}$ & 42 & 6 & 52 & Clay \\
\hline
\end{tabular}




\begin{tabular}{|c|c|c|c|c|c|c|c|c|c|c|}
\hline \multirow{2}{*}{$\begin{array}{c}\begin{array}{c}\text { Pedon } \\
\text { and } \\
\text { Horizon }\end{array} \\
\end{array}$} & \multirow{2}{*}{$\begin{array}{l}\text { Depth } \\
(\mathrm{cm})\end{array}$} & \multirow{2}{*}{$\begin{array}{l}\text { Colour } \\
\text { Moisture }\end{array}$} & \multirow{2}{*}{$\begin{array}{l}\text { Struc- } \\
\text { tures }\end{array}$} & \multicolumn{3}{|c|}{ Consistence } & \multirow{2}{*}{ Sand } & \multirow{2}{*}{ Silt } & \multirow{2}{*}{ Clay } & \multirow{2}{*}{$\begin{array}{c}\text { Texture } \\
\text { Class }\end{array}$} \\
\hline & & & & $\mathbf{w}$ & $\mathbf{m}$ & D & & & & \\
\hline \multicolumn{11}{|c|}{ P9 (Volcanic Hills) } \\
\hline Ap & $0-16$ & $7.5 \mathrm{YR} 3 / 2$ & $\mathrm{~m}, 3$, abk & vs, vp & vfi & $\mathrm{Vh}$ & 54 & 31 & 15 & Sandy Loam \\
\hline $\mathrm{Bw}$ & $16-34$ & $7.5 \mathrm{YR} 3 / 3$ & $\mathrm{~m}, 1$, abk & $\mathrm{s}, \mathrm{p}$ & fi & $\mathrm{H}$ & 42 & 24 & 34 & Clay Loam \\
\hline $\mathrm{Bt}$ & $34+$ & $7.5 \mathrm{YR} 4 / 4$ & $\mathrm{~m}, 1, \mathrm{abk}$ & ss. sp & fi & $\mathrm{H}$ & 50 & 15 & 35 & Sandy Clay \\
\hline \multicolumn{11}{|c|}{ P10 (Volcanic Hills) } \\
\hline Ap & $0-20$ & $7.5 \mathrm{YR} 3 / 3$ & $\mathrm{~m}, 3$, abk & ss, sp & fi & $\mathrm{H}$ & 48 & 26 & 26 & $\begin{array}{c}\text { Sandy Clay } \\
\text { Loam }\end{array}$ \\
\hline Bt1 & $20-44$ & 7.5 YR 3/4 & $\mathrm{m}, 3$, abk & $\mathrm{s}, \mathrm{p}$ & fi & $\mathrm{H}$ & 41 & 15 & 44 & Clay \\
\hline Bt2 & $44-76$ & $7.5 \mathrm{YR} 4 / 6$ & $\mathrm{c}, 3, \mathrm{sbk}$ & $\mathrm{s}, \mathrm{p}$ & fi & $\mathrm{H}$ & 42 & 14 & 44 & Clay \\
\hline $\mathrm{BC}$ & $76+$ & $5 \mathrm{YR} 4 / 6$ & $\mathrm{c}, 3, \mathrm{p}$ & vs, vp & vfi & $\mathrm{Vh}$ & 31 & 2 & 67 & Clay \\
\hline
\end{tabular}

Note. Structure: size: vf - very fine, $\mathrm{f}$ - fine, $\mathrm{m}$ - medium, c - coarse; structureless grade: 1 - weak, 2 - moderate, 3 strong; type: $\mathrm{cr}$ - crumb, sg - single grain, abk - angular blocky, sbk - sub-angular blocky. Consistence: $d r y$ (d): s - soft, 1 - loose, sh - slightly hard, h - hard, vh - very hard, eh - extremely hard; moist (m): 1 - loose, fr - friable, fi - firm, vfi very firm, efi - extremely firm, wet (w): so - non-sticky, ss - slightly sticky, s - sticky, vs - very sticky; po - non-plastic, ps - slightly plastic, $\mathrm{p}$ - plastic, vp - very plastic. 
Бюллетень Почвенного института им. В.В. Докучаева. 2021. Вып. 108

Dokuchaev Soil Bulletin, 2021, 108
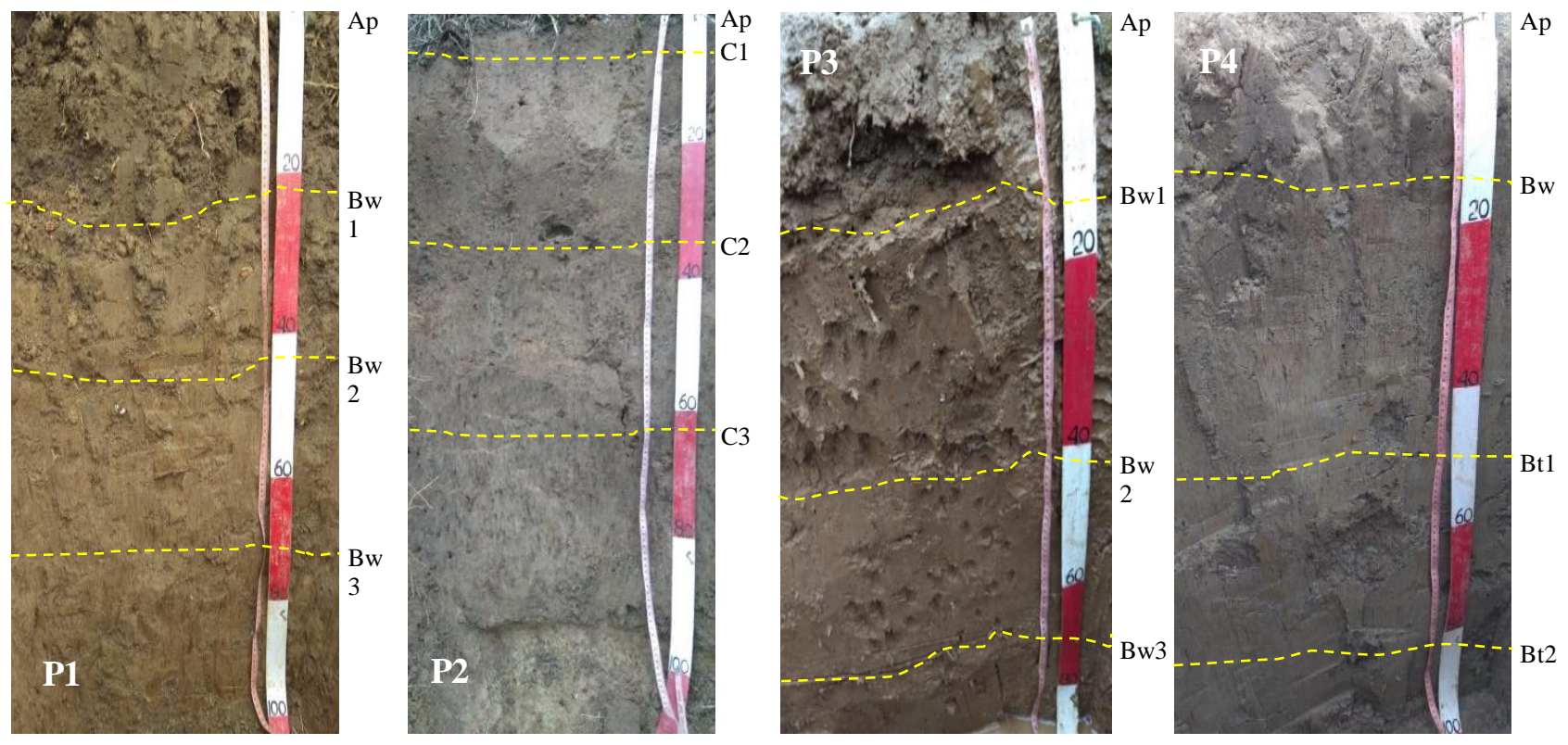

Fig. 2. Soil profile appearance of pedon 1 (P1), 2 (P2), 3 (P3) and pedon 4 (P4). 
While in P4, P6 and P7, soil colour varies from very dark grayish brown, dark brown to dark yellowish-brown with hue 10YR, values ranging from 3 to 4 , and chroma - from 2 to 6 . The colour of soil horizon $\mathrm{A}$ is darker than of horizon $\mathrm{B}$ due to the fact that the organic matter content in horizon A is higher than in horizon B (Yatno et al., 2015). The higher the organic matter content, the darker the soil colour is (Suharta, 2007). Soil colour seems to be a function of chemical and mineralogical composition (Swarnam et al., 2004; Walia, Rao, 1997), and the soil texture is influenced by topographic position and humidity regimes (Walia, Rao, 1997).

The soil structure varies from crumbs, angular blocky, sub angular blocky to prismatic, with the dominant angular blocky. P1, P3, P4, P6, P7, P8, P9 and P10 were mostly of angular blocky structure with the sizes varying from fine, medium to coarse, with weak and strong structural development. While the soil structures of P2 and P5 varies between crumbs, angular blocky, sub angular blocky and prismatic with the sizes ranging from fine, medium to coarse and the level of the soil structure development varying from weak, moderate to strong. The angular blocky soil structures were strongly associated with higher clay fractions (Devi et al., 2015). Crumbly soil structure indicates newly developed soil (Manik et al., 2017). Intensive soil tillage results in soil structure disturbance (Jambak et al., 2017). The variation of soil structure will be consistently affecting the soil.

The soil consistency in wet conditions varies between non-sticky and non-plastic on Pedon 2, slightly sticky and slightly plastic, sticky and plastic in the horizon on P1, P3, P4, P5, P6, P7, P8, P9 and P10, very sticky and very plastic on the surface horizon (Ap) of P9 and the subsurface horizon (BC) of P10, however, slightly sticky and slightly plastic consistencies prevail. While in moist conditions the consistencies vary from loose, firm to very firm, the firm consistencies are still dominant. P1, P3, P4, P5, P6, P7, P8, P9 and P10 were dominated by hard consistencies, while $\mathrm{P} 2$ - by loose ones. There is a very hard consistency in P1 and P10 in the subsurface horizon (Bw and BC), while in the surface horizon (Ap) a very hard consistency can be only found in P9. The consistency in dry conditions varies from loose, hard to very hard, with a dominant hard consistency. P1, P3, P4, P5, P6, P7, P8, P9 and P10 were dominated by a hard consistency, however, in P1 and 
P10 in the subsurface horizon (Bw3 and $\mathrm{BC}$ ) one could notice a very hard consistency, while in P9 precisely a very hard consistency was found on the surface horizon (Ap). Sticky and plastic consistency might form due to a high clay content in the soil (Sarkar et al., 2001); (Kadao et al., 2003), while non-sticky and non-plastic consistency might form due to a very little clay content (Sireesha, Naidu, 2015; Devi et al., 2015).

The soil texture of all pedons varies greatly between sandy clay loam, sandy loam, loamy sand, sandy loam, silty loam, clay loam, silty clay and clay, except for P7 that was of clay texture in all horizons. Wide variations of soil texture may be caused by the variations in parent material, topography, in-situ weathering, translocation of clays by eluviation and soil age (Satish et al., 2018). Apparently, the pedons located in the alluvial group were more dominated by clay fractions, although the distribution patterns were irregular. The distribution of irregular clay fractions was typical for sediment materials (Nurdin, 2010). While a sand fraction dominated the volcanic group, the distribution of sand and clay fractions shows the opposite pattern. A decrease in the sand fraction is due to the clay illuviation and in-situ mineral destruction process, which was characterized by a decline in the absolute amount of sand in the middle of the solum (Rachim, 1994). The texture that is found in the subsurface horizon is caused by higher weathering in the subsurface layer (Dutta, 2009). The clay content in the solum middles (B-illuviation) was higher than in the upper horizon (A-eluviation) and in the lower layer horizon. This indicates the occurrence of a lessivage process with some clay skins seen, so that an argillic horizon was formed in P4, P5, P8, P9 and P10. Although the process of eluviation and illuviation occurred, the pedon remained, but the clay skins were not found, so the cambic and candic horizons were formed.

\section{Soil chemical properties}

Soil chemical properties are presented in Table 3. Soil $\mathrm{pH}$ varies from acid ( $\mathrm{pH}$ 5.3) to neutral ( $\mathrm{pH}$ 7.2). The pedons located on the upper watershed or in volcanic groups (P2, P8, P9 and P10) have a lower $\mathrm{pH}$ than the ones located on the lower watershed or in alluvial groups ( $\mathrm{P} 1$, P3, P4, P5, P6, and P7). 
Table 3. Soil chemical properties in the Bulia micro watershed

\begin{tabular}{|c|c|c|c|c|c|c|c|c|c|c|}
\hline \multirow{3}{*}{$\begin{array}{l}\text { Pedon } \\
\text { and } \\
\text { Horizon }\end{array}$} & \multirow{3}{*}{$\begin{array}{c}\text { Depth } \\
\text { (cm) }\end{array}$} & \multicolumn{2}{|c|}{ pH 1:1 } & \multirow{3}{*}{$\begin{array}{l}\text { OC } \\
(\%)\end{array}$} & \multicolumn{4}{|c|}{ Exchangeable cations } & \multirow{3}{*}{ CEC } & \multirow{3}{*}{ BS $(\%)$} \\
\hline & & \multirow{2}{*}{$\mathrm{H}_{2} \mathrm{O}$} & \multirow{2}{*}{ KCl 1N } & & $\mathbf{K}$ & $\mathbf{N a}$ & $\mathbf{C a}$ & Mg & & \\
\hline & & & & & \multicolumn{4}{|c|}{$\left[\operatorname{cmol}(+) \mathrm{kg}^{-1}\right]$} & & \\
\hline \multicolumn{11}{|c|}{ P1 (Alluvial Plains) } \\
\hline Ap & $0-23$ & 6.20 & 5.40 & 2.29 & 0.29 & 1.88 & 18.02 & 1.86 & 42.40 & 52.00 \\
\hline Bw1 & $23-43$ & 6.00 & 5.20 & 0.90 & 0.18 & 2.01 & 15.47 & 2.97 & 39.19 & 52.62 \\
\hline Bw2 & $43-75$ & 6.40 & 5.50 & 0.74 & 0.14 & 2.19 & 12.36 & 2.50 & 37.85 & 45.42 \\
\hline Bw3 & $75-100$ & 6.50 & 5.60 & 0.65 & 0.14 & 2.23 & 15.22 & 3.26 & 37.58 & 55.49 \\
\hline \multicolumn{11}{|c|}{ P2 (Volcanic Mountains) } \\
\hline Ap & $0-5$ & 6.50 & 5.60 & 1.19 & 0.10 & 0.34 & 3.77 & 0.30 & 11.13 & 40.55 \\
\hline Bw1 & $5-37$ & 6.10 & 5.20 & 0.55 & 0.08 & 0.34 & 4.20 & 0.60 & 7.75 & 67.49 \\
\hline Bw2 & $37-61$ & 6.20 & 5.30 & 0.63 & 0.08 & 0.35 & 3.91 & 0.15 & 8.87 & 50.52 \\
\hline $\mathrm{C}$ & $61+$ & 6.20 & 5.30 & 0.56 & 0.08 & 0.36 & 6.51 & 0.76 & 12.29 & 62.79 \\
\hline \multicolumn{11}{|c|}{ P3 (Alluvial Plain) } \\
\hline $\mathrm{Ap}$ & $0-14$ & 5.60 & 4.60 & 0.80 & 0.05 & 1.46 & 4.84 & 0.61 & 16.15 & 43.00 \\
\hline Bw1 & $14-43$ & 6.00 & 5.10 & 0.41 & 0.09 & 1.57 & 7.72 & 1.70 & 19.78 & 56.00 \\
\hline Bw2 & $43-68$ & 5.80 & 4.90 & 0.32 & 0.05 & 1.55 & 8.95 & 0.91 & 21.58 & 53.00 \\
\hline $\mathrm{Bw} 3$ & $>68$ & 6.20 & 5.20 & 0.48 & 0.05 & 1.55 & 10.12 & 0.76 & 20.43 & 61.00 \\
\hline \multicolumn{11}{|c|}{ P4 (Alluvial Plains) } \\
\hline Ap & $0-14$ & 6.50 & 6.10 & 0.41 & 0.06 & 1.49 & 11.87 & 0.62 & 25.55 & 55.00 \\
\hline $\mathrm{Bw}$ & $14-50$ & 6.70 & 6.30 & 0.96 & 0.19 & 1.43 & 9.71 & 2.12 & 21.60 & 62.00 \\
\hline Bt1 & $50-81$ & 7.00 & 6.00 & 0.41 & 0.12 & 1.58 & 16.54 & 2.01 & 35.19 & 58.00 \\
\hline $\mathrm{Bt} 2$ & $81+$ & 5.80 & 5.00 & 0.24 & 0.09 & 1.56 & 13.47 & 6.66 & 33.04 & 66.00 \\
\hline
\end{tabular}




\begin{tabular}{|c|c|c|c|c|c|c|c|c|c|c|}
\hline \multirow{3}{*}{$\begin{array}{l}\text { Pedon } \\
\text { and } \\
\text { Horizon }\end{array}$} & \multirow{3}{*}{$\begin{array}{c}\text { Depth } \\
\text { (cm) }\end{array}$} & \multicolumn{2}{|c|}{ pH 1:1 } & \multirow{3}{*}{$\begin{array}{l}\text { OC } \\
(\%)\end{array}$} & \multicolumn{4}{|c|}{ Exchangeable cations } & \multirow{2}{*}{ CEC } & \multirow{3}{*}{ BS $(\%)$} \\
\hline & & \multirow{2}{*}{$\mathbf{H}_{2} \mathbf{O}$} & \multirow{2}{*}{ KCl 1N } & & $\mathbf{K}$ & $\mathbf{N a}$ & $\mathbf{C a}$ & Mg & & \\
\hline & & & & & \multicolumn{5}{|c|}{$\left[\mathrm{cmol}(+) \mathrm{kg}^{-1}\right]$} & \\
\hline \multicolumn{11}{|c|}{ P5 (Alluvial Plains) } \\
\hline Ap & $0-21$ & 5.20 & 4.50 & 0.08 & 0.16 & 2.29 & 9.39 & 2.93 & 28.39 & 52.00 \\
\hline $\mathrm{Bw}$ & $21-46$ & 5.10 & 4.30 & 0.49 & 0.20 & 2.30 & 8.62 & 3.08 & 28.40 & 50.00 \\
\hline Bt1 & $46-84$ & 5.70 & 4.90 & 0.57 & 0.12 & 0.26 & 7.69 & 2.00 & 21.56 & 47.00 \\
\hline $\mathrm{Bt} 2$ & $84-117$ & 5.10 & 4.20 & 0.50 & 0.17 & 0.30 & 10.38 & 2.05 & 31.34 & 41.00 \\
\hline Btg & $117+$ & 5.10 & 4.10 & 0.42 & 0.25 & 0.34 & 15.37 & 1.90 & 38.58 & 46.00 \\
\hline \multicolumn{11}{|c|}{ P6 (Alluvial Plains) } \\
\hline Ap & $0-12$ & 7.20 & 6.60 & 0.64 & 3.56 & 3.02 & 2.54 & 0.91 & 10.76 & 93.00 \\
\hline Bw1 & $12-34$ & 7.10 & 6.60 & 0.32 & 0.28 & 1.72 & 6.03 & 0.30 & 11.79 & 71.00 \\
\hline Bw2 & $34-71$ & 7.00 & 6.00 & 0.56 & 0.16 & 0.39 & 7.84 & 0.15 & 17.16 & 50.00 \\
\hline $\mathrm{C}$ & $71-90$ & 6.80 & 6.00 & 0.40 & 0.12 & 0.38 & 6.49 & 0.15 & 13.96 & 51.00 \\
\hline \multicolumn{11}{|c|}{ P7 (Alluvial Plains) } \\
\hline Ap & $0-6$ & 6.30 & 5.40 & 0.76 & 0.10 & 0.42 & 5.31 & 2.41 & 18.31 & 45.00 \\
\hline Bw1 & 6-17 & 6.10 & 5.10 & 0.57 & 0.09 & 0.39 & 6.77 & 2.15 & 22.99 & 41.00 \\
\hline Bw2 & $17-33$ & 5.90 & 5.00 & 0.57 & 0.07 & 0.44 & 8.55 & 4.04 & 25.45 & 51.00 \\
\hline $\mathrm{Bt}$ & $33-49$ & 5.60 & 4.80 & 0.33 & 0.08 & 0.45 & 13.64 & 3.72 & 29.79 & 60.00 \\
\hline $\mathrm{BC}$ & $49+$ & 5.90 & 5.00 & 0.41 & 0.12 & 2.04 & 13.16 & 0.31 & 33.06 & 47.00 \\
\hline
\end{tabular}




\begin{tabular}{|c|c|c|c|c|c|c|c|c|c|c|}
\hline \multirow{3}{*}{$\begin{array}{l}\text { Pedon } \\
\text { and } \\
\text { Horizon }\end{array}$} & \multirow{3}{*}{$\begin{array}{c}\text { Depth } \\
\text { (cm) }\end{array}$} & \multicolumn{2}{|c|}{ pH 1:1 } & \multirow{3}{*}{$\begin{array}{l}\text { OC } \\
(\%)\end{array}$} & \multicolumn{4}{|c|}{ Exchangeable cations } & \multirow{3}{*}{ CEC } & \multirow{3}{*}{ BS $(\%)$} \\
\hline & & \multirow{2}{*}{$\mathrm{H}_{2} \mathrm{O}$} & \multirow{2}{*}{ KCl 1N } & & $\mathbf{K}$ & $\mathrm{Na}$ & $\mathbf{C a}$ & Mg & & \\
\hline & & & & & \multicolumn{4}{|c|}{$\left[\mathrm{cmol}(+) \mathrm{kg}^{-1}\right]$} & & \\
\hline \multicolumn{11}{|c|}{ P8 (Volcanic Hills) } \\
\hline Ap & $0-7$ & 6.00 & 5.20 & 0.96 & 0.24 & 2.09 & 10.15 & 0.45 & 19.40 & 67.00 \\
\hline $\mathrm{Bw}$ & $7-24$ & 6.40 & 5.50 & 0.80 & 0.17 & 2.05 & 10.30 & 0.61 & 21.55 & 61.00 \\
\hline Bt1 & $24-44$ & 6.70 & 5.80 & 0.48 & 0.24 & 2.21 & 10.07 & 1.83 & 26.06 & 55.00 \\
\hline $\mathrm{Bt} 2$ & $44-63$ & 6.60 & 5.60 & 0.49 & 0.33 & 2.21 & 9.47 & 0.78 & 26.52 & 48.00 \\
\hline $\mathrm{Bt} 3$ & $63+$ & 6.60 & 5.60 & 0.50 & 0.49 & 2.35 & 13.84 & 1.27 & 32.83 & 55.00 \\
\hline \multicolumn{11}{|c|}{ P9 (Volcanic Hills) } \\
\hline Ap & $0-16$ & 5.30 & 4.50 & 0.80 & 0.13 & 2.15 & 10.15 & 0.61 & 24.80 & 53.00 \\
\hline $\mathrm{Bw}$ & $16-34$ & 6.20 & 5.30 & 0.57 & 0.10 & 2.30 & 12.44 & 0.77 & 29.51 & 53.00 \\
\hline $\mathrm{Bt}$ & $34+$ & 6.20 & 5.20 & 0.48 & 0.06 & 2.25 & 13.49 & 0.46 & 30.54 & 53.00 \\
\hline \multicolumn{11}{|c|}{ P10 (Volcanic Hills) } \\
\hline Ap & $0-20$ & 5.80 & 4.90 & 0.72 & 0.12 & 0.40 & 5.90 & 1.21 & 18.31 & 41.69 \\
\hline Bt1 & $20-44$ & 6.10 & 5.20 & 0.64 & 0.05 & 0.40 & 8.23 & 0.46 & 18.44 & 49.59 \\
\hline $\mathrm{Bt} 2$ & $44-76$ & 6.30 & 5.30 & 0.48 & 0.05 & 0.39 & 7.26 & 0.76 & 19.36 & 43.66 \\
\hline $\mathrm{BC}$ & $76+$ & 5.80 & 5.30 & 0.33 & 0.06 & 0.42 & 11.28 & 2.04 & 25.64 & 53.82 \\
\hline
\end{tabular}

Note. $\mathrm{CEC}$ - cation exchange capacity, $\mathrm{OC}$ - organic carbon, $\mathrm{BS}$ - base saturation. 

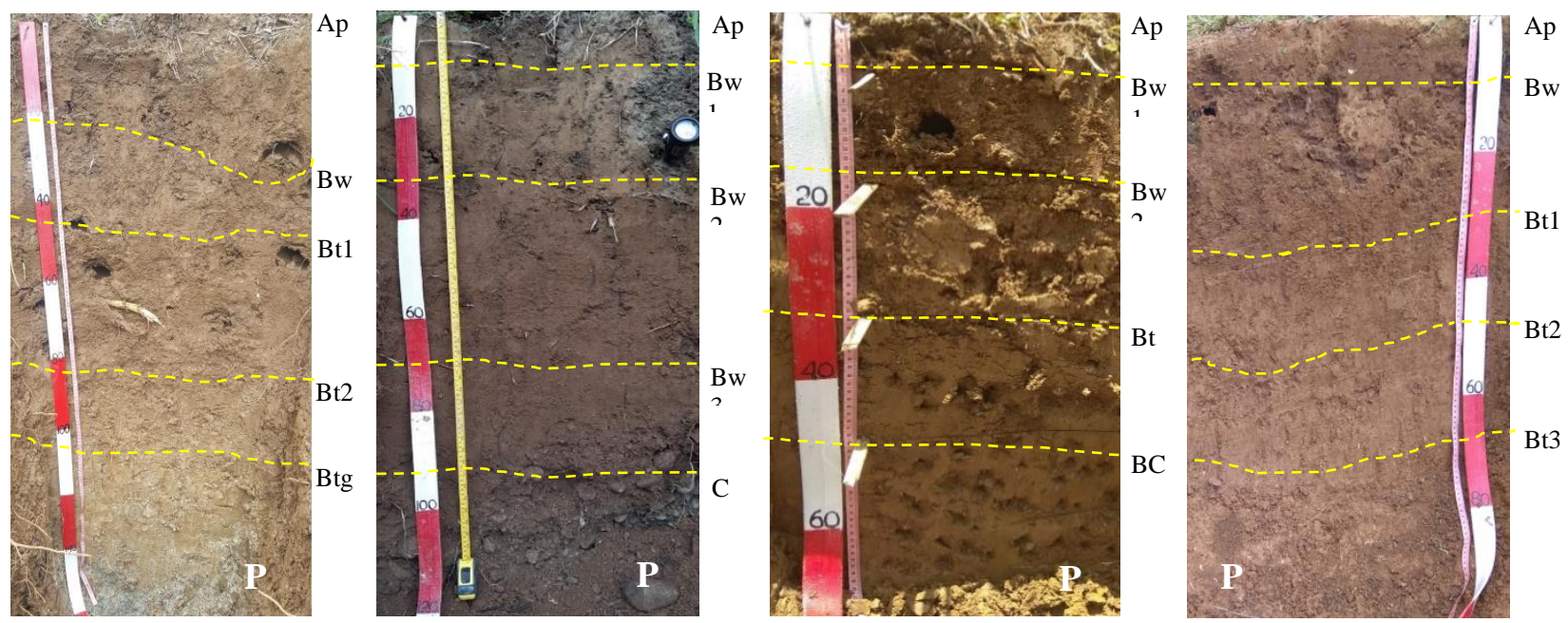

Fig. 3. Soil profile appearance of pedon 5 (P5), 6 (P6), 7 (P7) and pedon $8(\mathrm{P} 8)$. 
The pedon in the alluvial group represented a depressed area which is the accumulation of the bases carried by washing water from the hinterland section and the accumulation of more alkaline bases in soils with poorer drainage (Nurdin, 2010a). The pedons in the volcanic group experienced more intensive washing during the rain due to a better soil drainage. The trend of $\mathrm{pH}$ increasing with more depth may be explained by the release of organic acids during the decomposition of organic matter, these acids may have lowered the $\mathrm{pH}$ at the soil surface (Satish et al., 2018). The $\mathrm{pH}$ differences of $\mathrm{KCl}$ and the $\mathrm{pH}$ of $\mathrm{H}_{2} \mathrm{O}$ of all pedons show negative values. This means that all pedons are dominated by negatively charged clay minerals (Suharta, 2007). The acid and slightly acid soil $\mathrm{pH}$ values in P1, P3, P5, P9 and P10 indicated that the soil had developed but the level of soil development was not yet advanced, while the neutral soil $\mathrm{pH}$ in $\mathrm{P} 2, \mathrm{P} 4, \mathrm{P} 6, \mathrm{P} 7$, and $\mathrm{P} 8$ shows that the soil is relatively new.

Organic carbon (OC) varies from very low to high $(0.08-$ $2.28 \%$ ). The OC value was high in the surface horizon (Ap), except for $\mathrm{P} 4$ and P5. The OC value on the surface is higher due to the accumulation of organic materials, while its low values in P4 and P5 were due to the river flooding. The low $\mathrm{OC}$ value was also determined by a faster degradation of the organic material in the tropics and a low addition of farmyard manure (Vedadri, Naidu, 2018). A high OC distribution pattern on the surface and its dramatic decrease in horizon $\mathrm{B}$ in accordance with the depth is a general soil development pattern (Prasetyo, 2007).

The reserve (sum) of exchangeable bases vary between very low, low, medium, high and very high. Calcium cation is the dominating one in the exchangeable bases reserve, which ranges from 2.54-18.02 cmol $(+) \mathrm{kg}^{-1}$, while magnesium $\left(\mathrm{Mg}^{+}\right)$ranges from 0.15-6.66 cmol $(+) \mathrm{kg}^{-1}$, sodium $\left(\mathrm{Na}^{+}\right)$ranges from $0.26-3.02 \mathrm{cmol}(+) \mathrm{kg}^{-1}$, potassium $\left(\mathrm{K}^{+}\right)$ranges from $0.05-3.56 \mathrm{cmol}(+) \mathrm{kg}^{-1}$. Based on a number of bases, the P1, P5, P7, and P10 patterns follow the sequence: $\mathrm{Ca}^{2+}>$ $\mathrm{Mg}^{+}>\mathrm{Na}^{+}>\mathrm{K}^{+}$. This series pattern was the same as Nurdin $(\underline{2011})$ and Satish et al. (2018) reported. At the same time P2, P3, P4, P6, P8 and P9 follow the sequence: $\mathrm{Ca}^{2+}>\mathrm{Na}^{+}>\mathrm{Mg}^{+}>\mathrm{K}^{+}$. A high rate of exchangeable bases in the surface horizon (Ap) results from the fertilization during corn cultivation, while in the lower layer rainfall washing 
together with good drainage conditions make it possible to wash in the soil solum.

The cation exchange capacity (CEC) varies from low (7.7 cmol $\left.(+) \mathrm{kg}^{-1}\right)$ to very high $\left(42.40 \mathrm{cmol}(+) \mathrm{kg}^{-1}\right)$. The CEC is influenced by the levels of organic carbon and soil minerals (Prasetyo et al., 2007; Suharta, 2007). It seems that CEC in P1, P3, P4, P5, P7, P8, and P9 were more influenced by OC content than by soil minerals. The higher the soil OC, the higher the soil CEC is (Suharta, 2007). While P2, P6 and P10 were thought to be more influenced by soil minerals, these bases can be exchanged and this CEC will eventually affect base saturation. Base saturation (BS) varies from moderate $(40.55 \%)$ to very high $(93 \%)$. All pedons generally have a medium and a high BS, except P6, which has a very high BS on the surface horizon (Ap). The variations in BS may be caused by the variations in nature and/or the content of soil colloids, and a relatively high base saturation in the surface layers can be attributed to the recycling of cation base through vegetation (Devi, Kumar, 2010). In addition, if soil has a number of bases which are smaller than CEC, BS tends to be lower, whereas when the soil has a number of bases close to or higher than CEC, BS tends to be higher (Nurdin, 2010b).

Soil classification

Based on morphological and soil characteristics, the pedons are classified according to their family level and the orders of the soils found, namely Entisol, Inceptiol, Mollisol and Alfisol (Table 4, Figure 4). P1 and P7, which are located on a slope of 3\% and 5\%, are based on the molic epipedon with a $23 \mathrm{~cm}$ thickness and a cambic horizon. These pedons did not experience aquatic conditions at the depth of 50 $\mathrm{cm}$ from the soil surface (humustept), the soil colour had a value of 4 with a chroma of 2 or less and the soil structure was sub angular blocky. In addition, it had more clay content than the horizons below or above it. Based on these properties, P1 was classified as Typic Humustepts, fine, isohyperthermic.

$\mathrm{P} 2$, located on a slope of $15 \%$, is represented by the ochric epipedon with a $5 \mathrm{~cm}$ thickness and a candic horizon. In this pedon there was an increase in the percentage of clay in the fine soil fraction with a depth of $15 \mathrm{~cm}$ or less in the vertical distance. 
Бюллетень Почвенного института им. В.В. Докучаева. 2021. Вып. 108

Dokuchaev Soil Bulletin, 2021, 108

Table 4. Soil classification in the Bulia micro watershed

\begin{tabular}{|c|l|l|l|l|l|c|c|}
\hline \multirow{2}{*}{ Pedon } & \multicolumn{2}{|c|}{ Soil Classification } & \multicolumn{2}{c|}{ Area } \\
\cline { 2 - 8 } & \multirow{2}{*}{ Order } & \multicolumn{1}{|c|}{$\begin{array}{c}\text { Sub } \\
\text { Order }\end{array}$} & \multicolumn{1}{|c|}{$\begin{array}{c}\text { Great } \\
\text { Group }\end{array}$} & Sub Group & \multicolumn{1}{c|}{ Family } & ha & $\%$ \\
\hline P1 & Inceptisol & Ustept & Humustepts & $\begin{array}{l}\text { Typic } \\
\text { Humustepts }\end{array}$ & $\begin{array}{l}\text { Fine, isohypertermic, } \\
\text { Typic Humustepts }\end{array}$ & 596.88 & 8.53 \\
\hline P2 & Entisol & Psamment & $\begin{array}{l}\text { Ustipsamme } \\
\text { nts }\end{array}$ & $\begin{array}{l}\text { Typic } \\
\text { Ustipsamments }\end{array}$ & $\begin{array}{l}\text { Sandy, isohypertermic, } \\
\text { Typic Ustipsamments }\end{array}$ & 472.68 & 6.76 \\
\hline P3 & Inceptisol & Ustept & Humustepts & $\begin{array}{l}\text { Oxic } \\
\text { Humustepts }\end{array}$ & $\begin{array}{l}\text { Fine loamy, } \\
\text { isohypertermic, Oxic } \\
\text { Humustepts }\end{array}$ & $2,297.78$ & 32.86 \\
\hline P4 & Mollisol & Ustoll & Argiustolls & $\begin{array}{l}\text { Typic } \\
\text { Argiudolls }\end{array}$ & $\begin{array}{l}\text { Fine, isohypertermic, } \\
\text { Typic Argiustolls }\end{array}$ & 107.35 & 1.54 \\
\hline P5 & Alfisol & Ustalf & Paleustalfs & $\begin{array}{l}\text { Typic } \\
\text { Paleustalfs }\end{array}$ & $\begin{array}{l}\text { Fine loamy, } \\
\text { isohypertermic, Typic } \\
\text { Paleustalfs }\end{array}$ & $1,066.95$ & 15.26 \\
\hline P6 & Mollisol & Ustoll & Haplustolls & $\begin{array}{l}\text { Typic } \\
\text { Haplustolls }\end{array}$ & $\begin{array}{l}\text { Coarse loamy, } \\
\text { isohypertermic, Typic } \\
\text { Haplustolls }\end{array}$ & $1,026.23$ & 14.67 \\
\hline
\end{tabular}


Бюллетень Почвенного института им. В.В. Докучаева. 2021. Вып. 108

Dokuchaev Soil Bulletin, 2021, 108

\begin{tabular}{|c|c|c|c|c|c|c|c|}
\hline \multirow{2}{*}{ Pedon } & \multicolumn{5}{|c|}{ Soil Classification } & \multicolumn{2}{|c|}{ Area } \\
\hline & Order & $\begin{array}{l}\text { Sub } \\
\text { Order }\end{array}$ & $\begin{array}{l}\text { Great } \\
\text { Group }\end{array}$ & Sub Group & Family & ha & $\%$ \\
\hline P7 & Inceptisol & Ustept & Humustepts & $\begin{array}{l}\text { Typic } \\
\text { Humustepts }\end{array}$ & $\begin{array}{l}\text { Fine, isohypertermic, } \\
\text { Typic Humustepts }\end{array}$ & 248.4 & 3.55 \\
\hline P8 & Alfisol & Ustalf & Paleustalfs & $\begin{array}{l}\text { Ultic } \\
\text { Paleustalfs }\end{array}$ & $\begin{array}{l}\text { Fine loamy, } \\
\text { isohypertermic, Ultic } \\
\text { Paleustalfs }\end{array}$ & 61.87 & 0.88 \\
\hline P9 & Mollisol & Ustoll & Paleustolls & $\begin{array}{l}\text { Typic } \\
\text { Paleustolls }\end{array}$ & $\begin{array}{l}\text { Fine loamy, } \\
\text { isohypertermic, Typic } \\
\text { Paleustolls }\end{array}$ & 48.35 & 0.69 \\
\hline P10 & Alfisol & Ustalf & Haplustalfs & $\begin{array}{l}\text { Inceptic } \\
\text { Haplustalfs }\end{array}$ & $\begin{array}{l}\text { Fine, isohypertermic, } \\
\text { Inceptic Haplustalfs }\end{array}$ & $1,066.95$ & 15.26 \\
\hline \multicolumn{6}{|c|}{ Total } & $6,993.44$ & 100.00 \\
\hline
\end{tabular}



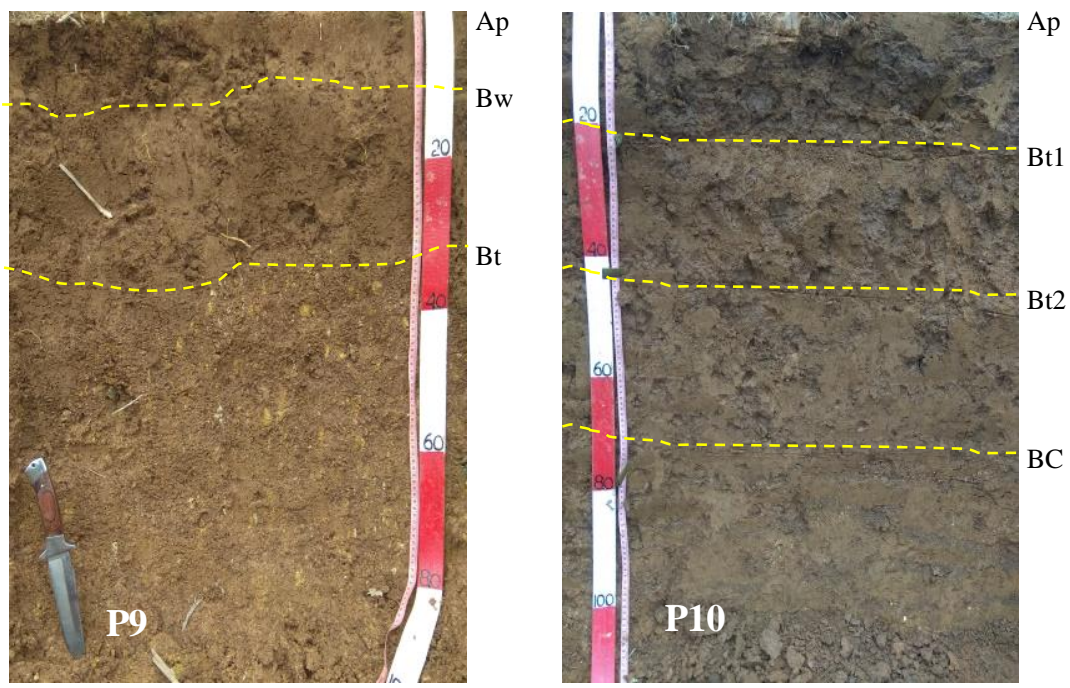

Fig. 4. Soil profile appearance of pedon 9 (P9), and pedon 10 (P10

The clay content was $4 \%$ or more (absolute value), which is more than that of the horizon above it, which has a total clay content in the soil fine fraction less than $20 \%$. In addition, it had a loamy sand texture (psamments) and a $\mathrm{CEC}$ value $\left(\mathrm{NH}_{4} \mathrm{OAc}, \mathrm{pH}\right.$ 7) $>16$ $\mathrm{cmol}(\mathrm{p}+) \mathrm{kg}^{-1}$. Based on these characteristics, P2 was classified as Typic Ustipsamments, sandy, isohyperthermic.

P3 is located on a slope of $1 \%$, represented by the umbric epipedon with a $14 \mathrm{~cm}$ thickness and a cambic horizon. This pedon did not experience aquatic conditions at the depth of $50 \mathrm{~cm}$ from the soil surface (humustept), the soil colour had a value of 4 with a chroma of 2 or less and the soil structure is sub angular blocky. In addition, it had more clay content than the horizons below or above and its CEC $\left(\mathrm{NH}_{4} \mathrm{OAc} 1 \mathrm{~N}\right.$ ) value was $17.96 \mathrm{cmol}(+) \mathrm{kg}^{-1}$ only (oxic). Based on these properties, P3 was classified as Oxic Humustepts, fine loamy, isohyperthermic.

P4 is located on a slope of $15 \%$, represented by the molic epipedon (BS 55\%) with a $14 \mathrm{~cm}$ thickness and an argillic horizon. This pe- 
don had a fine loamy class of particle size with a typical clay coating of the pore walls and the ped surface. In addition, there was $13 \%(>8 \%)$ of clay on the eluvial horizon and $10 \mathrm{YR}$ hue with a chroma $\leq 3$, and BS $>75 \%$. Based on these properties, P4 was classified as Typic Argiustolls, fine loamy, isohyperthermic.

P5 is located on a slope of 3\%, represented by the ochric epipedon with a $21 \mathrm{~cm}$ thickness and an argillic horizon. According to the particle-size classification, this pedon belongs to the fine loam and clay class with an argillic horizon thickness of $25 \mathrm{~cm}(>7.5 \mathrm{~cm})$, it also has a typical clay coating of the pore walls and the ped (aggregate) surface. In addition, the clay content in the argillic horizon is $64 \%$ or it contains 2.03 times more clay than the eluvial horizon, which was only $34 \%$, and 7.5 YR was typical of all horizons (paleustalf). Based on these properties, P5 was classified as Typic Paleustalfs, fine loamy, isohyperthermic.

P6 is located on a slope of 3\%, represented by the molic epipedon (BS 93\%) with a $12 \mathrm{~cm}$ thickness and a cambic horizon. This pedon had a sandy clay texture and does not have the combination of aquic conditions within $50 \mathrm{~cm}$ of the soil surface or artificial drainage (ustoll). The colour value was with a chroma $<6$ and it had a sub angular blocky soil structure. In addition, it had more clay content than the below horizons. Based on these properties, P6 was classified as Typic Haplustolls, coarse loamy, isohyperthermic.

P8 is located on a slope of 5\%, represented by the molic epipedon with a $7 \mathrm{~cm}$ thickness and an argillic horizon. According to the particle-size classification, this pedon belongs to the fine loam and fine clay class with an argillic horizon thickness of $20 \mathrm{~cm}(>7.5 \mathrm{~cm})$, also it has a typical clay coating of the pore walls and the ped surface. In addition, the clay content in the argillic horizon is $69 \%$ or it contains 2.65 times more clay than the eluvial horizon, which was only $26 \%$, and 7.5 YR hue was typical in all horizons (paleustalf), with BS of $61 \%$ or > $75 \%$ only (ultic). Based on these properties, P8 was classified as Ultic Paleustalfs, fine loamy, isohyperthermic.

P9 is located on an $8 \%$ slope, represented by the molic epipedon (BS 53\%) with a $7 \mathrm{~cm}$ thickness and an argillic horizon. According to the particle-size classification, this pedon belongs to the fine loam class with an argillic horizon thickness of $18 \mathrm{~cm}(>7.5 \mathrm{~cm})$, it also has a typ- 
ical clay coating of the pore walls and the ped surface. In addition, the clay content in the argillic horizon is $69 \%$ or it contains 4.60 times more clay than the eluvial horizon, which was only $18 \%$, and $7.5 \mathrm{YR}$ hue with a chroma of $\leq 4$, and BS of $<75 \%$ of all horizons (paleustoll). Based on these properties, P9 was classified as Typic Paleustolls, fine loamy, isohyperthermic.

P10 is located on a slope of $15 \%$, represented by the molic epipedon (BS 57\%, 50\%) with a $23 \mathrm{~cm}$ thickness and an argillic horizon. According to the particle-size classification, this pedon belongs to the fine loam and fine clay class with an argillic horizon thickness of 24 $\mathrm{cm}(>7.5 \mathrm{~cm})$, it also has a typical clay coating of the pore walls and the ped surface. In addition, the clay content in the argillic horizon is $88 \%$ or it contains 3.38 times more clay than the eluvial horizon, which was only $24 \%$, and 10 YR hue with a chroma of $\leq 3$, and BS $>75 \%$. Based on these properties, P10 was classified as Inceptic Haplustalfs, fine, isohyperthermic.

\section{Land quality classes}

The land quality (LQ) of the Bulia micro watershed was presented in Table 5 and Figure 5. The LQ of class II with the main factor determining the land stress was high temperature and low organic matter. The high temperature factor with an isohyperthermic soil temperature regime as an indicator was found on LU 3 (P3), while the low organic matter factor with an ochric epipedon indicator was only on LU 5 (P5). The LQ of class II is determined as good and this land has few problems for sustainable production, its productivity is generally very high and as a result, the response to management is high (Beinroth et al., 2001). Land management through the addition of organic matter, including green manure, may be adopted along with the recommended fertilizer doses (Sys et al., 1991; Singh et al., 2004; Mahaputra et al., 2019) and mulching to stabilize temperatures and maintain soil moisture (Odjugo, 2008; Eruola et al., 2012; Damaiyanti et al., 2013), which is of great importance. In addition, the use of rice straw mulch and sawdust mulch influences soil properties by decreasing the value of bulk density, increasing soil porosity and soil organic matter (Nasruddin, Hanum, 2015). 
Table 5. Land quality classes in the Bulia micro watershed

\begin{tabular}{|c|c|c|c|c|c|}
\hline \multirow{2}{*}{$\begin{array}{l}\text { Land } \\
\text { Unit }\end{array}$} & \multicolumn{2}{|c|}{ Land Quality } & \multirow{2}{*}{$\begin{array}{c}\text { Land } \\
\text { Quality } \\
\text { Class }\end{array}$} & \multicolumn{2}{|c|}{ Area } \\
\hline & $\begin{array}{l}\text { Major Land } \\
\text { Stress Factor }\end{array}$ & $\begin{array}{l}\text { Determinant of } \\
\text { Land Stress }\end{array}$ & & ha & $\%$ \\
\hline 3 & $\begin{array}{l}\text { High } \\
\text { temperatures }\end{array}$ & $\begin{array}{l}\text { Isohyperthermic of } \\
\text { soil temperature } \\
\text { regime }\end{array}$ & II & $2,297.78$ & 32.86 \\
\hline 5 & $\begin{array}{l}\text { Low organic } \\
\text { matter }\end{array}$ & Ochric epipedon & II & $1,066.95$ & 15.26 \\
\hline $\begin{array}{c}1,6 \\
7\end{array}$ & $\begin{array}{l}\text { Seasonal water } \\
\text { excess }\end{array}$ & Recent terraces & III & $1,871.51$ & 26.76 \\
\hline 2 & $\begin{array}{l}\text { Low structural } \\
\text { stability and/or } \\
\text { crusting }\end{array}$ & Entisols & IV & 472.68 & 6.76 \\
\hline 4 & $\begin{array}{l}\text { Low structural } \\
\text { stability and/or } \\
\text { crusting }\end{array}$ & Clay soils & IV & 107.35 & 1.54 \\
\hline $\begin{array}{c}8,9 \\
10\end{array}$ & $\begin{array}{l}\text { Low water } \\
\text { holding } \\
\text { capacity }\end{array}$ & Sandy clay loam & VI & 1177.17 & 16.83 \\
\hline \multicolumn{4}{|c|}{ Total } & $3,628.71$ & 51.89 \\
\hline
\end{tabular}

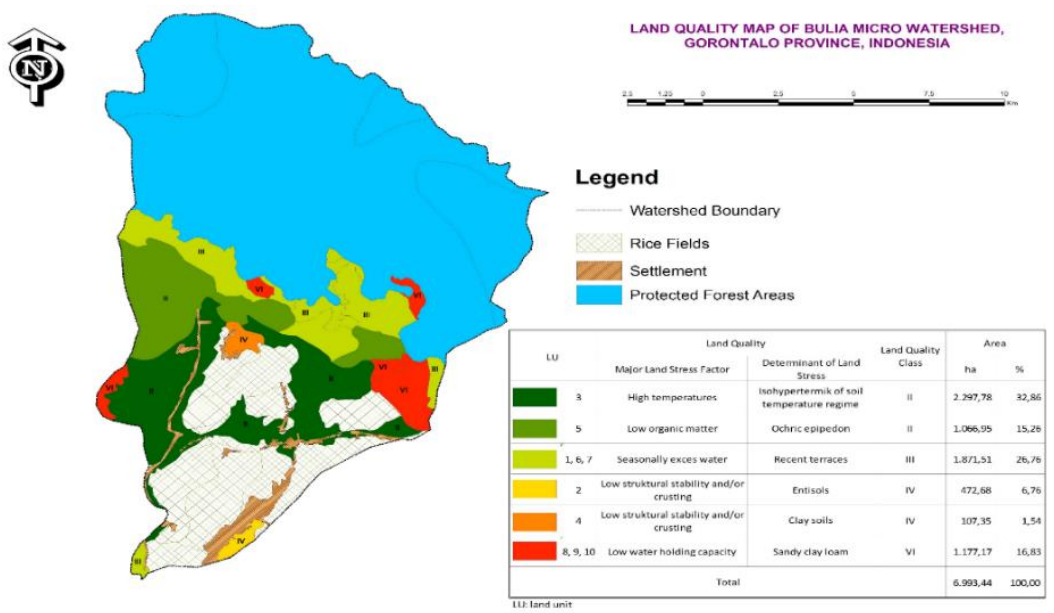

Fig. 5. Land quality map of the Bulia micro watershed. 
The LQ of class III with the main factor determining the land stress was a seasonal excess of water. The indicators determining the land stress in class III were new terraces spread over LU 1 (P1), LU 6 (P6) and LU 7 (P7). These new terraces are generally located around the border or river meanders which were prone to erosion and river bank landslides. The LQ of land class III was still considered good and this land has few problems for sustainable production, but has the higher risk of low input of corn production specifically, which results in a response to high management (Beinroth et al., 2001). The land management through the manufacture of gabions and riverbanks (Rahman, 2013), planting river bank reinforcement plants and terraces (Suyana et al., 2017) was of great importance. Bioengineering methods for river bank erosion control commonly used include planting bamboo (Noor et al., 2011). Fine plant roots play a significant role in increasing the shear strength of the soil (Ludwig et al., 2007). The effectiveness of plants in reducing erosion rate is influenced by (1) the canopy or plant canopy, (2) the organic material produced, (3) the root system and the ability of plants to cover the soil (Rachman et al., 2004). Vetiver plants were useful for stabilizing river banks, irrigation canals, river erosion control and coastal embankments, excavation slopes and embankments on highways, sand dunes, erosion on sloped agricultural land (Noor et al., 2011). Vetiver grass, which has strong fibrous roots, holds the ground (Susilawati, Veronika, 2016). Soil nailing was one of the most economical techniques for slopes stabilization of retaining walls because the system works quickly and does not require large space (Sinarta, 2014; Noor et al., 2011).

The LQ of class IV with the main factors determining land stress was low structural stability and/or crusting. These main factors consist of two indicators, namely clay soil and Entisol. The indicator of heavy clay (> 50\%) was scattered in LU 4 (P4). This indicator of clay soil was related to soil crusting which was shown by the average sub angular blocky soil structure, and the soil consistency under wet conditions was very sticky and very plastic, while it was very hard under dry conditions. Clay and organic matter are binding agents for aggregates (Rachim, 2007). Apparently, the influence of clay is more dominant than of organic matter as an aggregate binding agent because the OC content was very low. The Entisol indicators were spread on LU 2 (P2) 
only. Entisol is a soil that is still young and underdeveloped (Rachim, 2007). The results of the morphological analysis of the soil indicated that this Entisol has a granular structure (not solid), fine sized with a weak level of development, therefore the stability was relatively low. In addition, the texture of Entisol was classified as loamy sand with the sand of particle size which makes the soil structure stability relatively low. This class requires major inputs from conservation management; since a lack of plant nutrition is a major obstacle, therefore a plan to use good fertilizers should be adopted (Beinroth et al., 2001). Land management through the addition of organic matter can be applied together with recommended fertilizer doses (Sys et al., 1991; Singh et al., 2004; Mahaputra et al., 2019). Provision of organic matter in soils with clay texture can increase soil water content and available water capacity and reduce soil volume weight (Intara et al., 2011), increase soil porosity (Anastasia et al., 2014), increase N, P, and K uptake and crop yields in Entisol soils (Afandi et al., 2015). Addition of manure, compost and beneficial bacteria technology (custom bio) can reduce soil content weight, soil density, increase aggregate stability, soil porosity and moisture content in Entisol soil (Zulkarnain et al., 2013).

The LQ of class VI with the main factor determining the land stress was low water holding capacity. The indicators of the sandy clay loam texture were spread on LU 8 (P8), LU 9 (P9) and LU 10 (P10). The value of water available on the sandy clay loam texture was only around $20.42 \mathrm{~mm}$ (Haridjaja et al., 2013). The available water (pF) under conditions of field capacity ( $\mathrm{pF} 2.0)$ on LU 8, 9 and LU 10 was indicated as $34 \mathrm{~mm}, 38 \mathrm{~mm}$, and $37 \mathrm{~mm}$ respectively, and at permanent wilting conditions ( $\mathrm{pF} 4.2$ ) $24 \mathrm{~mm}, 17 \mathrm{~mm}$ and only $19 \mathrm{~mm}$ respectively, therefore, causing a low water holding capacity. This land should not be used for food production (i. e. corn) because this class requires major inputs from conservation management; since a lack of plant nutrition is a major obstacle, therefore a plan to use good fertilizers should be adopted or this land can be used as a biodiversity zone (Beinroth et al., 2001). However, if this land is used for agricultural cultivation, then the land management should consider the addition of organic matter along with the recommended fertilizer dosage (Sys et al., 1991; Singh et al., 2004; Mahaputra et al., 2019). Provision of organic material in soils with clay texture can increase soil water content 
and available water capacity and reduce soil volume weight (Intara et al., 2011), increase soil porosity (Anastasia et al., 2014).

\section{CONCLUSIONS}

Angular blocky, sticky, plastic consistencies, and hard consistencies prevailed in the soil structure of the Bulia micro watershed of Gorontalo Province, Indonesia. In the alluvial plain, a sand fraction prevailed in Volkan mountains. The soils in the Bulia micro watershed also react acid to neutral, with the range of very low to high OC levels, the reserve of exchangeable bases was dominated by $\mathrm{Ca}^{2+}$ in two series patterns, namely: $\mathrm{Ca}^{2+}>\mathrm{Mg}^{+}>\mathrm{Na}^{+}>\mathrm{K}^{+}$and $\mathrm{Ca}^{2+}>\mathrm{Na}^{+}>\mathrm{Mg}^{+}>\mathrm{K}^{+}$, $\mathrm{CEC}$ ranged from low to very high, and the base saturation varied from moderate to very high. The primary soils found were Entisol on P2 (Typic Ustipsamments), Inceptisol on P1 and P7 (Typic Humustepts), and P3 (Oxic Humustepts), Mollisol on P4 (Typic Argiudolls), P6 (Typic Haplustolls), and P9 (Typic Paleustolls), and Alfisol on P5 (Typic Paleustalfs), P8 (Ultic Paleustalfs) and P10 (Inceptic Haplustalfs). On the alluvial plains the land was categorized as the LQ class II, III and IV, the volcanic mountains were the LQ class IV, while the land on the volcanic hills was categorized as the LQ class VI. River bank erosion on the land river terraces can be held by the manufacture of gabions, talud, cliff reinforcement plants and terraces. The soil temperatures and high clay content can be regulated by mulching and organic materials.

\section{REFERENCES}

1. Afandi F.N., Siswanto B., Nuraini Y., Effect of giving various types of organic material on soil chemical properties on the growth and production of sweet potato plants in Entisol Ngrangkah Pawon, Kediri, Soil and Land Resources, 2015, Vol. 2(2), pp. 237-244, URL: http://jtsl.ub.ac.id.

2. Anastasia I., Izatti M., Suedy S.W.A., The effect of giving a combination of solid organic fertilizer and liquid organic on soil porosity and spinach plant growth (Amarantus tricolor L.), Biology Journal, 2014, Vol. 3(2), pp. 1-10.

3. Beinroth F.H., Eswaran H., Reich P.F., Global assessment of land quality, sustaining the global farm, Selected papers from the 10th International Soil Conservation Organization Meeting, 2001, pp. 569-574.

4. BPS Gorontalo Province, Gorontalo province in figure 2020, BPS 
Gorontalo Province, 2020, 1102001.75(75560.2004), pp. 1-535.

5. BPS Gorontalo Regency, Gorontalo regency in figure 2020, BPS Gorontalo Regency, 2020, 1102001.75(75020.2003), pp. 1-262.

6. Dutta M., Land use planning of Tipukjan watershed of Sivasagar district using GIS and remote sensing technique: Ph.D. Thesis, Assam Agricultural University, Jorhat, 2009.

7. Damaiyanti D.R.R., Aini N., Koesrihati, The study of organic mulch application on the growth and yield of red pepper (Capsicum annuum L.), Crop Production Journal, 2013, Vol. 1(2), pp. 25-32.

8. Devi P.A.V., Naidu M.V.S., Rao A.R., Characterization and classification of sugarcane growing soils in southern agro-climatic zone: A case study in eastern mandals of Chittoor district in Andhra Pradesh, Journal of the Indian Society of Soil Science, 2015, Vol. 63(3), pp. 245-258, DOI: 10.5958/09740228.2015.00034.1.

9. Eruola A.O., Bello N.J., Ufoegbune G.C., Makinde A.A., Effect of mulching on soil temperature and moisture regime on emergence, growth and yield of white yam in a tropical wet-and-dry climate, International Journal of Agriculture and Forestry, 2012, Vol. 2(1), pp. 93-100, DOI: 10.5923/j.ijaf.20120201.15.

10. Eviyati, Sulaeman, Analysis of soil, chemical, plants, water, and fertilizer, Indonesia Centre of Soil Research, 2009, pp. 1-203.

11. Husain J., Luntungan J.N., Kamagi Y.E.B., Nurdin B., Conservation-based corn farming model in Gorontalo Province. Research Report. Research, Development and Regional Environmental Impact Control Agency Gorontalo Province, Gorontalo, 2004.

12. Haridjaja O., Baskoro D.P.T., Setianingsih M., Different levels of field capacity by alhricks, free drainage, and pressure plate methods at different soil texture and relation for sunflower growth (Helianthus annuus L.), Soil Science and Environmental Journal, 2013, Vol. 15(2), 52 p, DOI: 10.29244/jitl.15.2.52-59.

13. Intara Y.I., Sapei A., Sembiring E.N., Djoefrie M.H.B., Affected of organic matter application at clay and clay loam soil texture on water holding capacity, Indonesian Agriculture Sciences Journal, 2011, Vol. 16(2), pp. 130135 .

14. Jambak M.K.F.A., Baskoro D.P.T., Wahjunie E.D., Characteristics of soil physic on soil conservation tillage system (Case study of Cikabayan Research Farm, Bogor), Soil and Land Bulletin, 2017, Vol. 1(1), pp. 44-50.

15. Jackson M.L., Soil chemical analysis, New Delhi: Prentice Hall of India (Pvt.) Ltd., 1973, 498 p.

16. Kadao S.H., Prasad J., Gajbhlye K.S., Characterization and classification of some typical banana growing soils of Wardha district of Maharashtra, 
Agropedology, 2003, Vol. 13(2), pp. 28-34.

17. Ludwig A., Bradford P., David B., Anton W., Vegetation patches and runoff-erosion as interacting ecohydrological processes in semiarid landscapes, Ecology, 2007, Vol. 86, pp. 288-297, DOI: 10.1890/03-0569.

18. Mahapatra S.K., Nagdev R., Gopal R., Surya J.N., Meena R.K., Yadav R.P., Singh S.K., Characterization and classification of the soils of Buraka micro-watershed in Haryana for integrated development, Journal of the Indian Society of Soil Science, 2019, Vol. 67(2), pp. 137-150, DOI: 10.5958/09740228.2019.00015.X.

19. Manik H., Marpaung P., Sabrina T., The level of soil development based on the pattern of clay mineral distribution in Subdistric Lumbanjulu, Distric of Toba Samosir, Agroteknologi FP USU, 2017, Vol. 5(2), pp. 422-433.

20. Nasruddin, Hanum H., Study of mulching effects on soil temperature, soil property, and growth of patchouli (Pogostemon cablin Benth), Floratek, 2015, Vol. 10, pp. 69-78.

21. Niranjana K.V., Ramamurthy V., Hegde R., Srinivas S., Koyal A., Naidu L.G.K., Sarkar D., Characterization, classification and suitability evaluation of banana growing soils of Pulivendla region. Andhra Pradesh, Journal of the Indian Society of Soil Science, 2011, Vol. 59(1), pp. 1-5, URL: https://www.indianjournals.com/ijor.aspx?target=ijor:jisss\&volume=59\&issue $=1$ \&article $=001$.

22. Noor A., Vahlevi J., Fathurrozi., Slope stabilization for erosion control with soil bioengineering using vetiver grass, Poros Teknik, 2011, Vol. 3(2), pp. 69-74.

23. Nurdin, Development, classification and rainfed paddy soils potency derived from lacustrine materials in paguyaman, gorontalo. MSc Thesis Bogor Agriculture University, 2010a. URL:

https://repository.ipb.ac.id/handle/123456789/57081.

24. Nurdin, The morfology, physics and soil chemistry of inceptisol derived from lacustrine paguyaman of gorontalo relating to soil managements, Jurnal Agroteknotropika, 2010b, Vol. 1(1), pp. 13-22.

25. Nurdin, Development and rainfed paddy soils potency derived from lacustrine material in paguyaman, Gorontalo, J. Trop. Soils, 2011, Vol. 16(3), pp. 267-278, DOI: $10.5400 /$ jts.2011.16.3.267.

26. Nurdin, Combination of soil conservation techniques and its effect on the yield of maize and soil erosion of dry land in biyonga sub-watershed, Gorontalo, J. Tek. Ling., 2012, Vol. 13(2), pp. 245-252.

27. Odjugo P.A.O., The effect of tillage systems and mulching on soil microclimate, growth and yield of yellow yam (Dioscorea cayenensis) in Midwestern Nigeria, African Journal of Biotechnology, 2008, Vol. 7(24), pp. 4500-4507, DOI: 10.4314/ajb.v7i24.59629. 
28. Oldeman L.R., Darmiyati S., An agroclimatic map of Sulawesi scale 1 2.500.000, Center Research for Agriculture, 1977, p. 1.

29. Puslitanak Research Team, Survey and mapping of semi-detailed soil resources (scale 1:50,000) in the North Sulawesi of Paguyaman area for the development of irrigation and sugarcane commodities. Final report. Book III Attachment of the Land Series. Center for Soil and Agro-climate Research, Agency for Agricultural Research and Development, Indonesian Ministry of Agriculture, Bogor, 1995.

30. Prasetyo B.H., Differentiation in Properties of vertisol from various parent materials, Indonesian Agriculture Sciences Journal, 2007, Vol. 9(1), pp. 2031.

31. Rachman A., Gantzer J., Anderson S.H., Soil hydraulic properties influenced by stiff-stemmed grass hedge systems, Soil Science Society of America Journal, 2004, Vol. 68(4), pp. 1386-1393, DOI: 10.2136/sssaj2004.1386.

32. Rachim D.A., Characterization os soils with low activity clay, and the effect of iron oxides on some soil properties, $\mathrm{PhD}$ thesis, Bogor Agricultural University, 1994, URL: https://repository.ipb.ac.id/handle/123456789/2463?show=full.

33. Rachim D.A., The foundamentals of soil genesis. Bogor: Departemen of Soil and Land Resources, Agriculture Faculty of Bogor Agriculture University, 2007, 41p.

34. Rahman A., Geographic information system model for estimating flow coefficient and its relationship with land cover in Riam Kanan River Basin of South Kalimantan Province, Bumi Lestari, 2013, Vol. 13(1), p. 1-8.

35. Sys C., Rast E.V., Debaveye J., Land evaluation. Part II. Principles in land evaluation and crop production calculations. International Training Centre for Post-Graduate Soil Scientists, University of Ghent, 1991.

36. Sarkar D., Gangipodhyay S.K., Velayutham M., Soil toposequence relationship and classification in lower outlier of Chhotanagpur plateau, Agropedology, 2001, Vol. 11, pp. 29-36.

37. Swarnam T.P., Velmurugan A., Rao Y.S., Characterization and classification of some soils from Shahibi basin in parts of Haryana and Delhi, Agropedology, 2004, Vol. 14, p. 114-122.

38. Singh C., Singh P., Singh R., Modern techniques of raising field crops, New Delhi: Oxford and IBH Publishing Co. Pvt. Ltd., 2004, pp. 1-583.

39. Satish S., Naidu M.V.S., Ramana K.V., Munaswamy V., Reddy G.P., Sudhakar P., Characterization and classification of the soils of Brahmanakotkur watershed in Kurnool district of Andhra Pradesh', Journal of the Indian Society of Soil Science, 2018, Vol. 66(4), pp. 351-361, DOI: 10.5958/0974-0228.2018.00044.0. 
40. Sinarta I., Methods of handling landslides with soil nailing', Paduraksa, 2014, Vol. 3(2), pp. 1-16.

41. Sireesha P.V.G., Naidu M.V.S., Clay mineralogy of soils developed from granite-gneiss of Kurnool District in Andhra Pradesh', Journal of the Indian Society of Soil Science, 2015, Vol. 63(1), pp. 16-23, DOI: 10.5958/09740228.2015.00003.1.

42. Soil survey manual, Soil Science Division Staff, United States Department of Agriculture (USDA), 2017, pp. 1-145, URL:

https://www.iec.cat/mapasols/DocuInteres/PDF/Llibre50.pdf.

43. Soil Survey Staff, Keys to soil taxonomy, Soil Conservation Service NRCS/USDA, 2014, Vol. 12, 360 p.

44. Subardja D., Sudarsono, The influence of land quality on productivity of maize in soils derived from volcanic and sedimentary rocks in the bogor area, Soil and Climate Journal, 2005, Vol. 23, pp. 38-46.

45. Suharta N., The characteristic of soil developed from felsic sediments in west kalimantan province and its implication to land management, Soil and Climate Journal, 2007, Vol. 25, pp. 11-26.

46. Sukarman S.R., Anda M., Suryani E., Guidelines for soil observation in the field, Agricultural Research and Development Agency, Ministry of Agriculture Republic of Indonesia, Bogor, 2017, pp. 1-149.

47. Supriya K., Naidu M.V.S., Kavitha P., Srinivasa M.R., Characterization, classification and evaluation of soils in semi-arid region of Mahanandi Mandal in Kurnool district of Andhra Pradesh', Journal of the Indian Society of Soil Science, 2019, Vol. 67(2), pp. 125-136, DOI: 10.5958/09740228.2019.00014.8.

48. Susilawati S., Veronika V., Vetiver grass study as a continuous slope safety, Civil Engineering Communication Media, 2016, Vol. 22(2), p. 99, DOI: $10.14710 / \mathrm{mkts} . v 22 \mathrm{i} 2.12886$.

49. Suyana J., Sumarno, Supriyono, Lestariningsih N.P., Mulching and strengthening of terraces in three types of plants against surface runoff, erosion, growth and yield of plants in Andisols, Agrosains, 2017, Vol. 19(1), p. 15, DOI: 10.20961/agsjpa.v19i1.20924.

50. Vedadri U., Naidu M.V.S., Characterization, classification and evaluation of soils in semi-arid ecosystem of Chillakur mandal in SPSR Nellore district of Andhra Pradesh, Journal of the Indian Society of Soil Science, 2018, Vol. 66 (1), pp. 9-19, DOI: 10.5958/0974-0228.2018.00002.6.

51. Walia C.S., Rao Y.S., Characteristics and classification of some soils of Trans-Yamuna plains, Journal of the Indian Society of Soil Science, 1997, Vol. 45(1), pp. 156-162.

52. Yasin M.G.H., Langgi W.F., White grain maize as an alternative for staple food, Iptek Tanaman Pangan, 2015, Vol. 9(2), pp. 108-117. 
53. Yatno E., Mulyanto B., Characteristics of soils formed from schist and their land suitability for cocoa crop in Kolaka and East Kolaka Regencies, Southeast Sulawesi, Soil and Climate Journal, 2016, Vol. 39(2), pp. 109-118. 54.Zulkarnain M., Prasetya B., Soemarno, Effects of compost, manure, and custom-bio on soil properties, growth and yield of sugarcane (Saccharum officinarum L.) in Entisol Kebun Ngrangkah of Pawon, Kediri, Indonesian Green Technology Journal, 2013, Vol. 2(1), pp. 45-52, URL: https://igtj.ub.ac.id/index.php/igtj/article/view/103. 